\title{
COMPARATIVE EVALUATION OF PROMISING LINE [(GIZA 83 X GIZA 80) X GIZA 89] X AUSTRALIAN AND LONG STAPLE COTTON GENOTYPES IN UPPER EGYPT
}

\author{
BADR, S. S. and S. A. EL TAHAN \\ Cotton Research Institute, Agricultural Research Center, Giza, Egypt.
}

(Manuscript received 18 February 2016)

\begin{abstract}
$\mathrm{T}$ he present investigation deals with comparison among some Egyptian cotton genotypes (Gossypium barbadense L.) with respect to yield and its components in four locations (Fayuom, Minia, Assuit and Sohag) in Upper Egypt during 2014 and 2015 seasons using different statistical analyses. Genotypes were G.80, G.90, (G.83x(G.75x5844))xG.80 and [(G.83xG.80)xG89] x Australum. A randomized complete block design was used in each location. The first step of comparative was analysis of individual locations. (G.83xG.80)xG.89]xAustralum significantly surpassed other genotypes in Fayuom $\left(L_{1}\right)$ in the two seasons except G90 in the 2014 season with respect to seed cotton yield. The results of lint cotton yield exhibited the same genotype was the best genotypes in Fayuom $\left(\mathrm{L}_{1}\right)$ in the two seasons and Minia $\left(L_{2}\right)$ in 2014 season. [G.83 x (G.75 x 5844)] x G.80 significantly exceeded other genotypes with respect to boll weight in Sohag $\left(L_{4}\right)$ in the first season. On the other hand, G80 significantly surpassed all genotypes with respect to the same trait in Fayuom $\left(L_{1}\right)$ and Sohag $\left(L_{4}\right)$ in the second season. [(G.83xG.80)xG.89]xAustralum significantly surpassed other genotypes with respect to lint percentage in three locations [ Fayuom $\left(\mathrm{L}_{1}\right)$, Minia $\left(\mathrm{L}_{2}\right)$ and Sohag $\left(\mathrm{L}_{4}\right)$ ] except [G.83 $\times(\mathrm{G} .75 \times$ 5844)] $\times$ G.80 in Sohag $\left(L_{4}\right)$ in the two seasons. In the two seasons, G.80 significantly surpassed other genotypes with respect to seed index in Sohag $\left(L_{4}\right)$ except G.90 in the first season. In the two seasons, non - significant variation due to genotypes was observed for lint index in all locations except Assuit $\left(\mathrm{L}_{3}\right)$ in the second season. Combined analysis was made to estimate the locations and the genotypes $x$ locations effects on cotton. The genotypes $x$ locations was non-significant with respect to yield and its components in the first season except seed cotton yield. On the contrary, the genotypes $x$ locations was significant with respect to lint cotton yield, boll weight and lint percentage in the second season. The third analysis was made to estimate the environments and genotypes $x$ environments effect on cotton. The interaction between genotypes $x$ environments was significant with respect to yield and its components except seed and lint index. The present study is very important for the regional program to evaluate the genotypes suitable for cultivation in Upper Egypt.
\end{abstract}

Key words: cotton, evaluation, locations . 


\section{INTRODUCTION}

Assumption basic to the analysis of variance requires that the all treatments have the variances (often called homoscedaticity), although the treatment or variety means may differ. Thus, the distribution of yield estimates for every plot should have the same variance. Generally, where there is no great deal of spread between the highest and the lowest means, the condition of unequality of variances is not likely to be serious. However, it sometimes happens that a control is included in an experiment that comprises several treatments. When the treatments effectively increase yield, there may be a greater or lesser degree of variability between the control yields than between those from the treated plots. In such cases the error variance may not be homogenous. This may be remedied by subdivision of the error term into homogenous components in order to test specific treatment comparisons. It should be clear that this assumption is very important, since the error term in the analysis of variance is a "pooled" error that comprises contributions from each of the treatments tested. When the magnitude of the experimental errors can be assumed to be the same for each treatment, a simple arithmetic mean can be taken to provide an estimate of treatment effect; a simple analysis of variance then would give the desired information. However, when the individual variances differ markedly, the analysis of variance is inappropriate. In order to determine whether this assumption is fulfilled, it is necessary to examine the variances for homogeneity. This may be done by the Bartlett test. Where the variances of the treatments are not homogeneous, it is sometimes possible to divide the sum of squares for error into components each of which is homogeneous, (LE Clerge et al. 1962).

Sing and Narayanan (2000) mentioned the benefits of applying randomized complete blocks design in plant breeding. The randomized complete block experiment is quite flexible. Since the variability between replications can be removed from the experimental error, it is unnecessary for the replications to be contiguous. An entire variable or replication may be omitted from the analysis when, for some reason, it is either lost or not comparable with the others components.

Abou Tour et al. (1996) evaluated five Egyptian cotton cultivars, viz., G.85, G.80, Dendera and G.75 at three locations in Upper Egypt ( Fayoum, Assuit and Sohag). Results revealed significant differences among cultivars with respect to lint cotton yield, seed index, lint percentage and fiber length in the individual locations. In contrast, non-significant variation due to cultivars was recorded for boll weight.

Awad et al. (2004) evaluated the two cultivars G90 and G83 with respect to yield and fiber properties in Upper Egypt (Assuit and Sohag). The results showed that G90 gave $5 \%$ higher yields (seed and lint) than G83.It slightly surpassed G83 for boll 
weight and gave the same range of lint percentage of G83. Fiber quality for G90 was nearly the same for the long staple cotton group in Upper Egypt.

Idris (2012) evaluated some Egyptian cotton genotypes in Upper Egypt using combined analysis. He found that homogeneity of variance test (Bartlett test) was not significant for boll weight, number of seeds per boll, seed index, lint index and lint percentage. In contrast, Bartlett test was significant for yields (seed and lint).

Researchers need a statistical measure to compare among genotypes under different locations and environments. The final goal was to study the interaction effect on cotton.

\section{MATERIALS AND METHODS}

Four long staple Egyptian cotton genotypes (Gossypium barbadense L.) were evaluated in four locations [ Fayuom $\left(L_{1}\right)$, Minia $\left(L_{2}\right)$, Assuit $\left(L_{3}\right)$ and Sohag $\left(L_{4}\right)$ ] in Upper Egypt during two seasons (2014 and 2015). Two of genotypes were cultivars, viz., G.80 and G90. The two remainders were (Bulk families), viz., [G83 x (G75 x 5844)] x G80 and [(G83xG80)xG89]xAustralum.

A randomized complete block design with four replications was used in each location. Planting was during the last week of March. All agricultural practices were done as usual.

Genotypes were evaluated for seed cotton yield (SCY) and lint cotton yield (LCY) in kentar / feddan ( $\mathrm{k} / \mathrm{fed}$ ). One sample (50 bolls) was obtained from each plot to estimate boll weight (BW) in g., seed index (SI) in g., lint index (LI) in g., and lint percentage (LP).

\section{Statistical analysis}

The first analysis was traditional (individual locations). Statistical analyses were straightforward according to Cochran and Cox (1950), Federer (1955), Gomez and Gomez (1984), Roger (1994) and Mcpherson (2001). The differentiate of genotypes means were compared by L.S.D. test as given by Steel and Torrie (1980). All comparisons were done at 0.05 level of significance.

Homogeneity test of variances (Bartlett test) was used according to procedures reported by Bailey (1994) before starting the combined analysis. The combined randomized complete block design was carried out with the data of genotypes in four locations to estimate the locations and environments effects on genotypes, (Table 1). Statistical analysis was straightforward according to Mcintosh (1983). The genotypes means were compared by least significant difference (L.S.D.) test as given by Steel and Torrie (1980). All comparisons were done at 0.05 level of significance. 
Table 1. Different analyses for cotton evaluation .

\begin{tabular}{|c|c|c|c|}
\hline \multicolumn{2}{|c|}{ Individual seasons } & \multicolumn{2}{|c|}{ Two seasons } \\
\hline Source of variation & $\mathrm{df}$ & Source of variation & $\mathrm{df}$ \\
\hline Locations (L) & $\mid-1$ & Environments ( $\mathrm{E}$ ) & e - 1 \\
\hline Replications / (L) & $I(r-1)$ & Replications / (E) & e $(r-1)$ \\
\hline Genotypes (G) & $(g-1)$ & Genotypes ( G ) & $(g-1)$ \\
\hline$G \times L$ & $(g-1)(I-1)$ & $G \times E$ & $(g-1)(e-1)$ \\
\hline Experimental error & $I(r-1)(g-1)$ & Experimental error & $e(r-1)(g-1)$ \\
\hline Total & Irg- 1 & Total & erg- 1 \\
\hline
\end{tabular}

\section{RESULTS AND DISCUSSION}

The analysis of randomized complete block design was carried out with the data of the individual locations as previously mentioned in the two successive seasons to estimate genotypes variance within locations, (Table 2).

In the two seasons, significant variation due to genotypes was observed for seed and lint cotton yield in the two locations Fayuom $\left(L_{1}\right)$ and Minia $\left(L_{2}\right)$.

The results of seed cotton yield showed [(G83xG80)xG89]xAustralum significantly surpassed all other genotypes in Fayuom $\left(L_{1}\right)$ in the two seasons except G90 in the 2014 season.

In combined analysis of the two seasons, non-significant differences among genotypes in Minia $\left(L_{2}\right)$ except G80 for seed cotton yield was observed, (Table 4).

The results of lint cotton yield revealed that genotype [(G83xG80)xG89]xAustralum was the best genotypes in Fayuom $\left(L_{1}\right)$ in the two seasons and Minia $\left(L_{2}\right)$ in 2014 season, it maintained the first rank, significantly surpassed other genotypes except [G83 x (G75 x 5844)] x G80 in Minia $\left(L_{2}\right)$ in the 2014 season. In the second season, no significant difference among genotypes in Minia $\left(L_{2}\right)$ except G80 for seed cotton yield was observed, (Table 4).

In 2014 season, non-significant variation due to genotypes was recorded for boll weight in all locations except Sohag $\left(L_{4}\right)$, (Table 2). [G83 x (G75 x 5844)] x G80 significantly exceeded other genotypes with respect to boll weight in Sohag $\left(L_{4}\right)$. In contrast, significant variation due to genotypes was observed for boll weight in all locations except Minia ( $\left.L_{2}\right)$ in 2015 seasons, (Table 2). G.80 significantly surpassed all genotypes with respect to the same trait in Fayuom $\left(L_{1}\right)$ and Sohag $\left(L_{4}\right)$. No significant difference among genotypes in Assuit ( $\left.L_{3}\right)$ except G.80 for boll weight was observed, (Table 4). 
In both 2014 and 2015 seasons, significant variation due to genotypes was observed for lint percentage except in Assuit $\left(L_{3}\right)$ in the first season, (Table 2). In the two seasons, [(G.83xG.80)xG.89]xAustralum significantly surpassed other genotypes with respect to lint percentage in three locations [ Fayuom $\left(L_{1}\right)$, Minia $\left(L_{2}\right)$ and Sohag $\left(L_{4}\right)$ ] except [G.83 x (G.75 x 5844)] x G.80 in Sohag $\left(L_{4}\right)$. No significant differernce among genotypes in Assuit $\left(L_{3}\right)$ except G80 for lint percentage in the second season was observed, (Table 4).

In the two seasons, non-significant variation due to genotypes was recorded for seed index except Fayuom $\left(L_{1}\right)$ in the first season and Sohag $\left(L_{4}\right)$ in the two seasons, (Table 2). In 2014 season, no significant difference among genotypes in Fayuom $\left(L_{1}\right)$ except [(G.83xG.80)xG.89]xAustralum. In the two seasons, $G 80$ significantly surpassed other genotypes with respect to seed index in Sohag $\left(L_{4}\right)$ except G90in the first season, (Table 4).

In both 2014 and 2015 seasons, no significant variation due to genotypes was observed for lint index in all locations except in Assuit $\left(L_{3}\right)$ in the second season, (Table 2). G.80 significantly exceeded other genotypes in Assuit $\left(L_{3}\right)$ except [G.83 x (G.75 x 5844)] x G.80, (Table 4).

The combined randomized complete block design was carried with the data of the four locations with respect to individual seasons to estimate the locations effects on genotypes, (Table 1).

Significant differences among locations were observed for yield and its components in individual seasons indicated that these traits were affected by locations. In addition, the analysis of combined analysis exhibited the interaction between genotypes $x$ locations was non-significant with respect to yield and its components in the first season except seed cotton yield. On the contrary, the interaction between genotypes $x$ locations was significant with respect to lint cotton yield, boll weight and lint percentage in the second season, (Table 3).

The combined randomized complete block design was carried with the data of the four locations with respect to two seasons to estimate the environmental effects on genotypes, (Table 1).

Significant differences among environments were observed for yield and its components in individual seasons indicating that these traits were affected by environments. On the other hand, the analysis of combined analysis revealed that the interaction between genotypes $x$ environments was significant with respect to yield and its components except seed and lint index, (Table 3). 
Table 2. Mean squares of traits for individual locations

\begin{tabular}{|c|c|c|c|c|c|c|c|c|c|}
\hline \multirow[b]{3}{*}{$\begin{array}{l}\text { Source of } \\
\text { variation }\end{array}$} & \multirow[b]{3}{*}{$\mathrm{df}$} & \multicolumn{8}{|c|}{ Seed cotton yield (k/fed.) } \\
\hline & & \multicolumn{4}{|c|}{2014 Season } & \multicolumn{4}{|c|}{2015 Season } \\
\hline & & $\mathrm{L}_{1}$ & $\mathrm{~L}_{2}$ & $\mathrm{~L}_{3}$ & $L_{4}$ & $\mathrm{~L}_{1}$ & $\mathrm{~L}_{2}$ & $\mathrm{~L}_{3}$ & $L_{4}$ \\
\hline Replications & 3 & 0.075 & 1.89 & 0.510 & 7.79* & 3.49 & 1.17 & 0.536 & 1.85 \\
\hline Genotypes & 3 & $6.35 * *$ & $3.91 * *$ & 3.04 & 1.41 & $9.14 * *$ & $12.55^{*}$ & 3.70 & 14.38 \\
\hline $\begin{array}{l}\text { Experimental } \\
\text { error }\end{array}$ & 9 & 0.814 & 0.513 & 0.890 & 1.90 & 0.994 & 2.63 & 1.45 & 9.86 \\
\hline Total & 15 & & & & & & & & \\
\hline \multirow[b]{2}{*}{$\begin{array}{l}\text { Source of } \\
\text { variation }\end{array}$} & \multirow[b]{2}{*}{$\mathrm{df}$} & \multicolumn{8}{|c|}{ Lint cotton yield (k/fed.) } \\
\hline & & $\mathrm{L}_{1}$ & $\mathrm{~L}_{2}$ & $\mathrm{~L}_{3}$ & $L_{4}$ & $\mathrm{~L}_{1}$ & $\mathrm{~L}_{2}$ & $\mathrm{~L}_{3}$ & $L_{4}$ \\
\hline Replications & 3 & 0.077 & 2.65 & 0.627 & $14.11^{*}$ & 5.46 & 1.55 & 0.661 & 3.46 \\
\hline Genotypes & 3 & $13.17^{* *}$ & $6.94 * *$ & 4.59 & 1.37 & $19.57^{* *}$ & $18.59 *$ & 7.08 & 29.51 \\
\hline $\begin{array}{l}\text { Experimental } \\
\text { error }\end{array}$ & 9 & 1.42 & 0.809 & 1.56 & 3.38 & 1.56 & 3.89 & 2.38 & 15.07 \\
\hline Total & 15 & & & & & & & & \\
\hline \multirow[b]{2}{*}{$\begin{array}{l}\text { Source of } \\
\text { variation }\end{array}$} & \multirow[b]{2}{*}{ df } & \multicolumn{8}{|c|}{ Boll weight $(\mathrm{g})$} \\
\hline & & $\mathrm{L}_{1}$ & $\mathrm{~L}_{2}$ & $\mathrm{~L}_{3}$ & $\mathrm{~L}_{4}$ & $\mathrm{~L}_{1}$ & $\mathrm{~L}_{2}$ & $\mathrm{~L}_{3}$ & $L_{4}$ \\
\hline Replications & 3 & 0.080 & 0.011 & 0.014 & 0.005 & 0.003 & 0.009 & $0.018 *$ & 0.008 \\
\hline Genotypes & 3 & 0.016 & 0.060 & 0.012 & $0.060 * *$ & $0.068 * *$ & 0.031 & $0.025 *$ & $0.196 * *$ \\
\hline $\begin{array}{l}\text { Experimental } \\
\text { error }\end{array}$ & 9 & 0.045 & 0.020 & 0.006 & 0.003 & 0.002 & 0.021 & 0.004 & 0.005 \\
\hline Total & 15 & & & & & & & & \\
\hline \multirow[b]{2}{*}{$\begin{array}{l}\text { Source of } \\
\text { variation }\end{array}$} & \multirow[b]{2}{*}{ df } & \multicolumn{8}{|c|}{ Lint percentage } \\
\hline & & $\mathrm{L}_{1}$ & $\mathrm{~L}_{2}$ & $\mathrm{~L}_{3}$ & $\mathrm{~L}_{4}$ & $\mathrm{~L}_{1}$ & $\mathrm{~L}_{2}$ & $L_{3}$ & $L_{4}$ \\
\hline Replications & 3 & 0.047 & 0.607 & 0.079 & 0.534 & 0.721 & 0.330 & 0.113 & 0.122 \\
\hline Genotypes & 3 & $6.27 * *$ & $4.61 *$ & 1.70 & $2.484 * *$ & $1.82 * *$ & $1.96 * *$ & $2.29 *$ & $3.03 * *$ \\
\hline $\begin{array}{l}\text { Experimental } \\
\text { error }\end{array}$ & 9 & 0.221 & 0.798 & 0.682 & 0.325 & 0.212 & 0.221 & 0.435 & 0.171 \\
\hline Total & 15 & & & & & & & & \\
\hline \multirow[b]{2}{*}{$\begin{array}{l}\text { Source of } \\
\text { variation }\end{array}$} & \multirow[b]{2}{*}{$\mathrm{df}$} & \multicolumn{8}{|c|}{ Seed index $(\mathrm{g})$} \\
\hline & & $\mathrm{L}_{1}$ & $\mathrm{~L}_{2}$ & $\mathrm{~L}_{3}$ & $\mathrm{~L}_{4}$ & $\mathrm{~L}_{1}$ & $\mathrm{~L}_{2}$ & $\mathrm{~L}_{3}$ & $\mathrm{~L}_{4}$ \\
\hline Replications & 3 & 0.191 & 0.287 & 0.397 & 0.282 & 0.305 & 0.775 & 0.244 & 0.118 \\
\hline Genotypes & 3 & $1.68 * *$ & 0.429 & 0.511 & $0.835^{* *}$ & 0.331 & 0.585 & 0.405 & $1.29 * *$ \\
\hline $\begin{array}{l}\text { Experimental } \\
\text { error }\end{array}$ & 9 & 0.207 & 0.488 & 0.240 & 0.081 & 0.255 & 0.247 & 0.144 & 0.075 \\
\hline Total & 15 & & & & & & & & \\
\hline \multirow[b]{2}{*}{$\begin{array}{l}\text { Source of } \\
\text { variation }\end{array}$} & \multirow[b]{2}{*}{$\mathrm{df}$} & \multicolumn{8}{|c|}{ Lint index (g) } \\
\hline & & $\mathrm{L}_{1}$ & $\mathrm{~L}_{2}$ & $\mathrm{~L}_{3}$ & $\mathrm{~L}_{4}$ & $\mathrm{~L}_{1}$ & $\mathrm{~L}_{2}$ & $\mathrm{~L}_{3}$ & $L_{4}$ \\
\hline Replications & 3 & 0.088 & 0.059 & 0.099 & 0.132 & 0.090 & $0.264 *$ & 0.090 & 0.083 \\
\hline Genotypes & 3 & 0.122 & 0.177 & 0.064 & 0.043 & 0.110 & 0.147 & $0.548^{*}$ & 0.097 \\
\hline $\begin{array}{l}\text { Experimental } \\
\text { error }\end{array}$ & 9 & 0.129 & 0.121 & 0.080 & 0.075 & 0.107 & 0.067 & 0.107 & 0.075 \\
\hline Total & 15 & & & & & & & & \\
\hline
\end{tabular}

$*$, ** Significant at 0.05 and 0.01 levels, respectively. 
Table 3. Mean squares of traits for individual and two seasons.

\begin{tabular}{|c|c|c|c|c|c|c|c|}
\hline \multirow[b]{3}{*}{ Source of variation } & \multirow[b]{3}{*}{ df } & \multicolumn{6}{|c|}{ Individual seasons } \\
\hline & & \multicolumn{6}{|c|}{2014 Season } \\
\hline & & SCY & LCY & BW & LP & SI & LI \\
\hline Locations (L) & 3 & $173.29 * *$ & $244.85^{* *}$ & $1.57 * *$ & $15.15^{* *}$ & $6.83 * *$ & $2.56^{* *}$ \\
\hline Replications / (L) & 12 & 2.56 & 4.37 & 0.027 & 0.317 & 0.289 & 0.095 \\
\hline Genotypes (G) & 3 & $7.60 * *$ & $14.86^{* *}$ & 0.037 & $13.14 * *$ & $2.94 * *$ & $0.332 *$ \\
\hline Gx L & 9 & $2.37 *$ & 3.74 & 0.037 & 0.638 & 0.171 & 0.024 \\
\hline Experimental error & 36 & 1.03 & 1.79 & 0.018 & 0.506 & 0.254 & 0.101 \\
\hline Total & 63 & \multicolumn{6}{|c|}{2015 Season } \\
\hline Source of variation & df & SCY & LCY & BW & LP & SI & LI \\
\hline Locations (L) & 3 & $259.44 * *$ & $447.89 * *$ & $1.37 * *$ & $20.25^{* *}$ & $4.02 * *$ & $1.90 * *$ \\
\hline Replications / (L) & 12 & 1.76 & 2.78 & 0.010 & 0.322 & 0.360 & 0.132 \\
\hline Genotypes (G) & 3 & $16.39 *$ & $33.05^{* *}$ & $0.070 * *$ & $5.89 * *$ & $1.84 * *$ & $0.522 * *$ \\
\hline Gx $\mathrm{L}$ & 9 & 7.79 & $13.90 *$ & $0.083^{* *}$ & $1.07 * *$ & 0.256 & 0.126 \\
\hline Experimental error & 36 & 3.73 & 5.72 & 0.008 & 0.260 & 0.180 & 0.089 \\
\hline Total & 63 & \multicolumn{6}{|c|}{ Two seasons } \\
\hline Source of variation & $\mathrm{df}$ & SCY & LCY & BW & LP & SI & LI \\
\hline Environments ( E ) & 7 & $205.15^{* *}$ & $328.86 * *$ & $1.62 * *$ & $16.26 * *$ & $6.87 * *$ & $2.60 * *$ \\
\hline Replications / (E) & 24 & 2.16 & 3.58 & 0.019 & 0.319 & 0.325 & 0.113 \\
\hline Genotypes ( G ) & 3 & $17.51 * *$ & $37.41 * *$ & $0.071 * *$ & $17.14 * *$ & $4.71 * *$ & $0.776 * *$ \\
\hline $\mathrm{G} \times \mathrm{E}$ & 21 & $5.28 * *$ & $9.06 * *$ & $0.057 * *$ & $1.00 * *$ & 0.193 & 0.076 \\
\hline Experimental error & 72 & 2.38 & 3.76 & 0.013 & 0.383 & 0.217 & 0.095 \\
\hline Total & 127 & & & & & & \\
\hline
\end{tabular}

*, ** Significant at 0.05 and 0.01 levels, respectively. 
Table 4. Mean of yield and its components.

\begin{tabular}{|c|c|c|c|c|c|c|c|c|}
\hline \multirow[b]{3}{*}{ Genotypes } & \multicolumn{8}{|c|}{ Seed cotton yield (k/fed.) } \\
\hline & \multicolumn{4}{|c|}{2014 Season } & \multicolumn{4}{|c|}{2015 Season } \\
\hline & $\mathrm{L}_{1}$ & $\mathrm{~L}_{2}$ & $\mathrm{~L}_{3}$ & $\mathrm{~L}_{4}$ & $\mathrm{~L}_{1}$ & $\mathrm{~L}_{2}$ & $\mathrm{~L}_{3}$ & $\mathrm{~L}_{4}$ \\
\hline G80 & 5.22 & 3.29 & 6.87 & 11.91 & 12.71 & 3.58 & 7.81 & 11.49 \\
\hline G90 & 6.62 & 4.36 & 9.00 & 13.12 & 11.79 & 5.62 & 5.58 & 13.34 \\
\hline [G83x(G75x5844)]xG80 & 5.35 & 5.34 & 8.06 & 12.16 & 11.41 & 7.73 & 7.35 & 15.62 \\
\hline [(G83xG80)xG89]xAustralum & 7.91 & 5.39 & 8.04 & 11.83 & 14.79 & 6.69 & 6.95 & 15.22 \\
\hline LSD & 1.44 & 1.14 & ---- & ---- & 1.59 & 2.59 & $\begin{array}{ll}--- \\
\end{array}$ & ---- \\
\hline LSD $(G \times L)$ & \multicolumn{4}{|c|}{0.73} & \multicolumn{4}{|c|}{----} \\
\hline $\mathrm{LSD}(\mathrm{G} \times \mathrm{E})$ & \multicolumn{8}{|c|}{0.77} \\
\hline \multirow[b]{2}{*}{ Genotypes } & \multicolumn{8}{|c|}{ Lint cotton yield (k/fed.) } \\
\hline & $\mathrm{L}_{1}$ & $\mathrm{~L}_{2}$ & $\mathrm{~L}_{3}$ & $\mathrm{~L}_{4}$ & $\mathrm{~L}_{1}$ & $\mathrm{~L}_{2}$ & $\mathrm{~L}_{3}$ & $\mathrm{~L}_{4}$ \\
\hline G80 & 6.66 & 4.21 & 8.88 & 14.44 & 16.58 & 4.50 & 9.74 & 14.01 \\
\hline G90 & 8.15 & 5.43 & 11.43 & 15.82 & 15.10 & 6.82 & 6.65 & 16.41 \\
\hline [G83x(G75x5844)]xG80 & 6.76 & 6.70 & 10.36 & 14.83 & 14.91 & 9.45 & 9.08 & 19.85 \\
\hline [(G83xG80)xG89]xAustralum & 10.56 & 7.12 & 10.70 & 14.89 & 19.69 & 8.41 & 8.57 & 19.42 \\
\hline LSD & 1.90 & 1.44 & ---- & ----- & 2.00 & 3.15 & ---- & ----- \\
\hline LSD $(G \times L)$ & \multicolumn{4}{|c|}{-.-- } & \multicolumn{4}{|c|}{1.72} \\
\hline $\operatorname{LSD}(G \times E)$ & \multicolumn{8}{|c|}{0.97} \\
\hline \multirow[b]{2}{*}{ Genotypes } & \multicolumn{8}{|c|}{ Boll weight $(\mathrm{g})$} \\
\hline & $\mathrm{L}_{1}$ & $\mathrm{~L}_{2}$ & $\mathrm{~L}_{3}$ & $\mathrm{~L}_{4}$ & $\mathrm{~L}_{1}$ & $\mathrm{~L}_{2}$ & $\mathrm{~L}_{3}$ & $\mathrm{~L}_{4}$ \\
\hline G80 & 2.80 & 2.48 & 2.80 & 3.01 & 3.25 & 2.81 & 2.70 & 3.70 \\
\hline G90 & 2.87 & 2.22 & 2.74 & 2.93 & 3.13 & 2.66 & 2.84 & 3.46 \\
\hline [G83x(G75x5844)]xG80 & 2.89 & 2.24 & 2.87 & 3.20 & 3.16 & 2.86 & 2.87 & 3.27 \\
\hline [(G83xG80)xG89]xAustralum & 2.95 & 2.27 & 2.80 & 2.97 & 2.94 & 2.82 & 2.85 & 3.20 \\
\hline LSD & ---- & $\begin{array}{ll}--- \\
-\end{array}$ & $\begin{array}{ll}--- \\
-\end{array}$ & 0.09 & 0.07 & $\begin{array}{ll}--- \\
\end{array}$ & 0.10 & 0.11 \\
\hline $\mathrm{LSD}(\mathrm{G} \times \mathrm{L})$ & \multicolumn{4}{|c|}{---- } & \multicolumn{4}{|c|}{0.06} \\
\hline \multirow[t]{3}{*}{$\operatorname{LSD}(G \times E)$} & & & & & & & & \\
\hline & & & & Lint $p e$ & entage & & & \\
\hline & $\mathrm{L}_{1}$ & $\mathrm{~L}_{2}$ & $\mathrm{~L}_{3}$ & $\mathrm{~L}_{4}$ & $\mathrm{~L}_{1}$ & $\mathrm{~L}_{2}$ & $\mathrm{~L}_{3}$ & $\mathrm{~L}_{4}$ \\
\hline G80 & 40.5 & 40.5 & 41.0 & 38.3 & 41.4 & 39.9 & 39.5 & 38.9 \\
\hline G90 & 39.4 & 39.5 & 40.8 & 38.4 & 40.7 & 38.6 & 37.8 & 39.0 \\
\hline [G83x(G75x5844)]xG80 & 40.1 & 40.0 & 41.0 & 39.2 & 41.5 & 38.9 & 39.2 & 40.4 \\
\hline [(G83xG80)xG89]xAustralum & 42.4 & 42.0 & 42.2 & 40.0 & 42.3 & 40.0 & 39.1 & 40.6 \\
\hline LSD & 0.8 & 1.4 & ---- & 0.9 & 0.7 & 0.8 & 1.1 & 0.7 \\
\hline LSD $(G \times L)$ & & & & & & & & \\
\hline $\operatorname{LSD}(G \times E)$ & & & & & & & & \\
\hline & & & & Seed i & ex (g) & & & \\
\hline Genotypes & $\mathrm{L}_{1}$ & $\mathrm{~L}_{2}$ & $\mathrm{~L}_{3}$ & $L_{4}$ & $\mathrm{~L}_{1}$ & $\mathrm{~L}_{2}$ & $\mathrm{~L}_{3}$ & $\mathrm{~L}_{4}$ \\
\hline G80 & 10.39 & 9.25 & 10.25 & 10.82 & 10.26 & 10.08 & 11.42 & 11.50 \\
\hline G90 & 10.44 & 8.94 & 9.94 & 10.67 & 10.23 & 10.35 & 10.85 & 11.01 \\
\hline [G83x(G75x5844)]xG80 & 10.33 & 8.81 & 9.73 & 10.22 & 10.43 & 9.92 & 10.96 & 10.59 \\
\hline$[(\mathrm{G} 83 \times \mathrm{G} 80) \times \mathrm{G} 89] \times$ Australum & 9.10 & 8.46 & 9.40 & 9.81 & 9.76 & 9.44 & 10.68 & 10.19 \\
\hline LSD & 0.73 & ---- & ---- & 0.45 & ---- & ---- & ---- & 0.44 \\
\hline LSD $(G \times L)$ & & & & & & & & \\
\hline LSD $(G \times E)$ & & & & & & & & \\
\hline & & & & Lint i & ex (g) & & & \\
\hline Genotypes & $\mathrm{L}_{1}$ & $\mathrm{~L}_{2}$ & $\mathrm{~L}_{3}$ & $\mathrm{~L}_{4}$ & $\mathrm{~L}_{1}$ & $\mathrm{~L}_{2}$ & $\mathrm{~L}_{3}$ & $\mathrm{~L}_{4}$ \\
\hline G80 & 7.08 & 6.29 & 6.98 & 6.73 & 7.25 & 6.68 & 7.47 & 7.33 \\
\hline G90 & 6.73 & 5.83 & 6.77 & 6.57 & 7.00 & 6.51 & 6.59 & 7.08 \\
\hline [G83x(G75x5844)]xG80 & 6.81 & 5.87 & 6.68 & 6.49 & 7.40 & 6.30 & 7.06 & 7.17 \\
\hline [(G83xG80)xG89]xAustralum & 6.69 & 5.93 & 6.87 & 6.54 & 7.16 & 6.27 & 6.86 & 6.96 \\
\hline LSD & ---- & ---- & ---- & ---- & $\begin{array}{ll}--- \\
\end{array}$ & $\begin{array}{ll}--- \\
\end{array}$ & 0.52 & $\begin{array}{ll}--- \\
\end{array}$ \\
\hline LSD $(G \times L)$ & & & & & & & & \\
\hline LSD $(G \times E)$ & & & & & & & & \\
\hline
\end{tabular}

--: Not significant at .05 level. 


\section{REFERENCES}

1. Awad, H.Y., Awaad M.M., Mohamed S.A., El Adly H.H., El Ameen T.M., Mohamed A.A. and Eissa A.E. 2004. Giza 90, a new, long staple Egyptian cotton variety for Middle and Upper Egypt. Egyptain Journal of Agricultural Research Center, 82 (3) 1349-1361.

2. Abou Tour, H. B., Seyam S. M. and Abd El Rahman L. M. 1996. Analytical study on the economic characters of new and commercial Egyptian cotton. Egypt. J. Agric. Res., 74 (3) : 781-791.

3. Idris, H.A. 2012. Comparison between some cultivars and varieties of Egyptian cotton using different analysis of latin square design. Bull. Fac. Agric., Cairo Univ., 63 (1): $19-28$.

4. Bailey, N. T. 1994. Statistical Methods in Biology. Third Edit. Cambridge University Press, Cambridge, England.

5. Cochran, W.G. and G. M. Cox. 1950. Experimental Designs. John Wiley and Sons, New York., U.S.A.

6. Federer, W. T. 1955. Experimental Design. Theory and Application. The Macmilan Company New York, U.S.A.

7. Gomez, K. A. and A. A. Gomez. 1984. Statistical Procedures for Agricultural Research. Wiley, New York, U.S.A.

8. Le Clerge, E. L., Leonard W. H. and Clark A.G. 1962. Field Plot Techniques. Burgess publishing company, New York, U.S.A.

9. Mcintosh, M. S. 1983. Analysis of combined experiments. Agron. J. 75: 153-155.

10. Mcpherson, G. 2001. Applying and Interpreting Statistics A Comprehensive Guide. Second Edit., Springer Verlag, New York, Inc. U.S.A.

11. Roger G. P. 1994. Agricultural Field Experiments Design and Analysis. Marcel Dekker, Inc. New York, U.S.A.

12. Sing, P., and Narayanan S. S. 2000. Biometrical Techniques in Plant BreedinG Second Edit., Ludhiana, New Delhi, India.

13. Steel, R. G. and Torrie J. H. 1980. Principles and Procedures of Statistics. Second Edit., McGraw. Hill. Book Co. New York, U.S.A. 


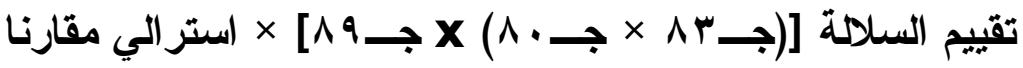

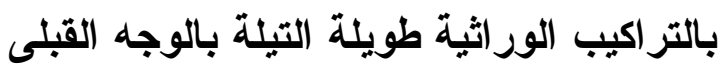

\author{
سامى سعد بلر ، سعيد عبد الرازق الطحان
}

$$
\text { معهد بحوث القطن - مركز البحوث الزراعية - الجيزة - مصر }
$$

تم تقييم المحصول ومكوناته لأربع نز اكيب ور اثية من القطن المصرى من طبقة الاقطان طويلة

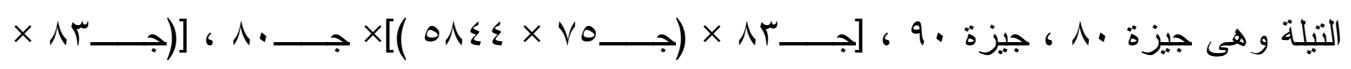

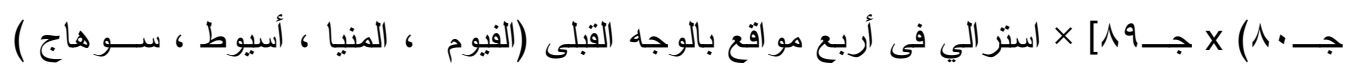

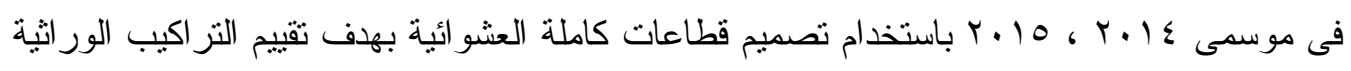
الجديدة .

أجرى التحليل الاحصائى بالنسبة لكل موقع على حده خلال الموسمين وفق الأسـس المعروفــة لتحليل قطاعات الكاملة العشو ائية.

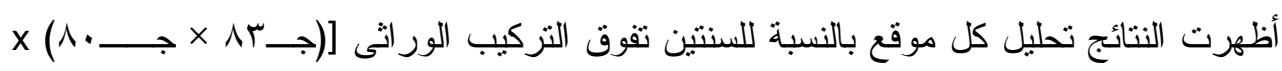
جـ1^] × استر الي معنويا فى صفة محصول القطن الزهر على جميع التز اكيب الور اثثية في الفيــوم فى السنتين ماعدا جيزة · 9 خلال الموسم الأول.

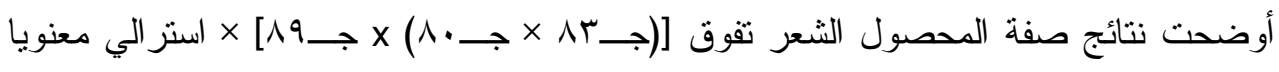
فى الفيوم خلد مد الدراسة بالاضاف إلى تفوقه فى المنيا فى الموسم الاول.

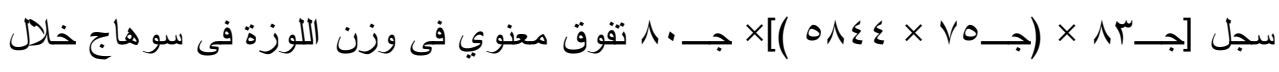

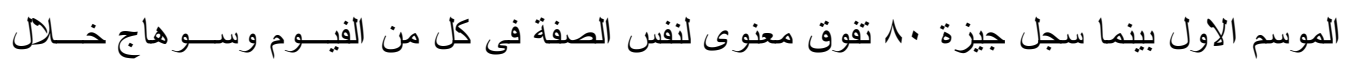

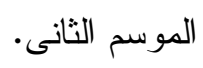

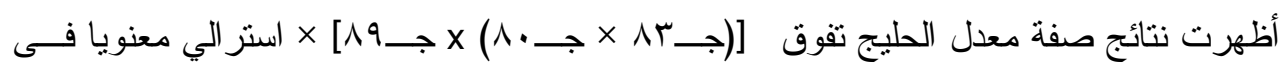

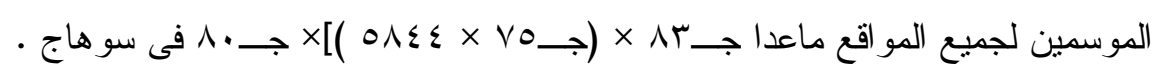

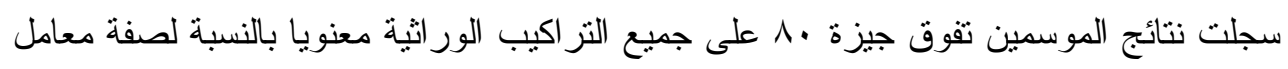

$$
\text { البذرة فى سو هاج ماعدا جيزة • 9 فى الموسم الأول. }
$$

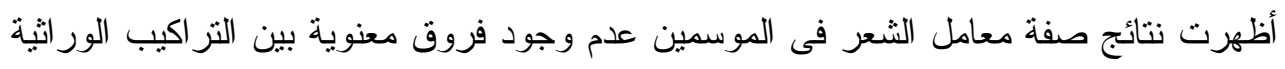

$$
\text { فى جميع المو اقع ماعدا أسيوط فى الموسم الثانى. نقائ. }
$$

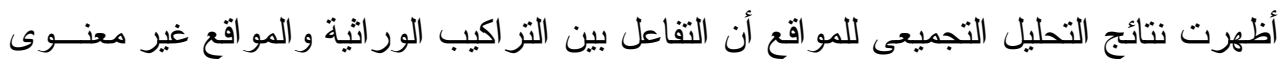

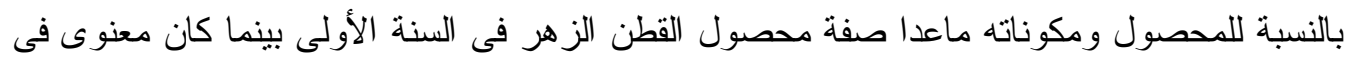

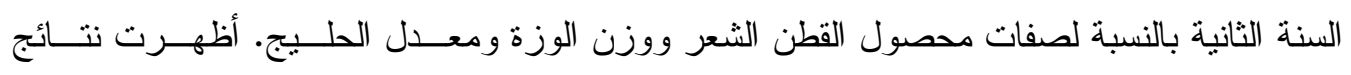

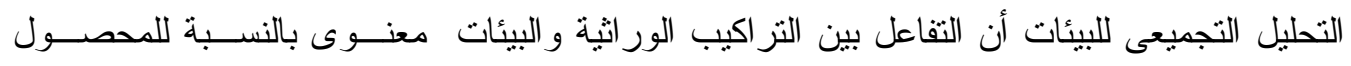
ومكوناته ماعدا صفة معامل البذرة و الشعر . ويستفاد من هذه الدراسة فى بر امج التقييم الاقليمى للتز اكيب الور اثية. 